Dieter Birnbacher

\title{
Kommentar zu Gaertner/Schwettmann: Untersuchungen zur Einschätzung von Gerechtigkeit
}

\begin{abstract}
The changes in the meaning of social justice described by Gaertner and Schwettmann are interpreted as a shift of emphasis within a relatively constant family of meanings. It is argued that any workable concept of social justice is the product of a balancing of a number of different principles of justice that are strictly incompatible and easily come into conflict with one another. In response to changing economic and cultural conditions certain members of the family are given priority without completely abandoning the other members. A parallel is drawn with the changes in the conceptions of justice operative in the distribution of scarce organ transplants.
\end{abstract}

Der 1950 verstorbene Philosoph Nicolai Hartmann gehörte zu der bereits zu seiner Zeit kleinen Zahl von Denkern, die meinten, Werte schwebten als eine Art platonischer Ideen über den Köpfen der wertenden Menschen. Selbst radikale Veränderungen in den gesellschaftlichen und individuellen Wertungen sollten der objektiven Realität und Unwandelbarkeit der Werte nichts anhaben können. Während sich die Wertungen wandeln, sollten die Werte selbst konstant bleiben. $\mathrm{Zu}$ den unterschiedlichen Wertungen komme es lediglich durch die wechselnden Perspektiven, mit der unterschiedliche Individuen, Gesellschaften, Kulturen und Zeiten ihren Blick auf den ,Werthimmel' richten: Wie die Sterne sich sie je nach Standort und Jahreszeit dem Auge in wechselnden Ausschnitten und Anordnungen darbieten, blieben auch die Werte trotz wechselnder Wertungsperspektiven dieselben. Auch wenn sie lange Zeit unbeachtet bleiben, stünden sie doch jederzeit bereit, in späteren Zeiten erneut in ein dann aktuelles Wertsystem aufgenommen zu werden.

Vielleicht ist Hartmanns, Werthimmel', seiner metaphysischen Voraussetzungen entkleidet, gar kein so schlechtes Bild für die von den Untersuchungen von Gaertner und Schwettmann nahegelegten Wandlungen des Gerechtigkeitsbegriffs. Auch wenn wir uns angewöhnt haben, von ,der' Gerechtigkeit zu sprechen, ist Gerechtigkeit kein homogener Wert, sondern ein mehrdimensionales Spektrum von Werten, weniger ein einzelner Stern am Werthimmel als vielmehr ein Sternnebel mit verschwommenen Strukturen und unscharfen Rändern. Obwohl die Werte in diesem Nebel bestimmte Gemeinsamkeiten aufweisen - z.B. die, dass sie alle in irgendeiner Form mit ,Gleichheit' zu tun haben -, sind sie 
nur begrenzt miteinander kompatibel und lassen sich nur selten so kombinieren, dass die Synthese ein konsistentes Ganzes ergibt. Entsprechend ,leerformelhaft sind Appelle an Gerechtigkeit, wenn sie offen lassen, welche spezifischen Ausprägungen und Varianten von Gerechtigkeit damit angezielt sind.

Die Metapher vom Werthimmel, der sich selbst gleich bleibt, aber aus verschiedenen Perspektiven betrachtet jeweils anders erscheint, ist für das Verständnis der Wandlungen in den Gerechtigkeitsvorstellungen deshalb hilfreich, weil es nur wenige aus der Tradition bekannte Gerechtigkeitsbegriffe zu geben scheint, die endgültig verabschiedet worden sind und in den gegenwärtig vorfindlichen Einstellungen gar nicht mehr auftauchen. Selbst dezidiert ,antiquierte ${ }^{6}$ Vorstellungen, etwa von einer Verteilung von Gütern oder Diensten nach dem Kriterium des gesellschaftlichen Rangs, überleben in gesellschaftlichen Nischen wie der Etikette und dem diplomatischen Protokoll. Die meisten in der Tradition entwickelten Gerechtigkeitsbegriffe existieren weiter und werden im Prinzip auch dann akzeptiert, wenn sie in der individuellen und gesellschaftlichen Praxis keine Rolle mehr spielen. Je nach wechselndem Zeitgeist und wechselnder gesellschaftlicher Stimmungslage werden bestimmte Gerechtigkeitskriterien ausgeblendet und eingeblendet, aber nicht gänzlich ausgelöscht. Die ausgeblendeten geraten nicht so weit in Vergessenheit, dass sie nicht mehr verstanden werden. Sie werden lediglich nur so nachrangig berücksichtigt, dass sie für die in der Praxis anstehenden Entscheidungen wirkungslos bleiben.

Es ist z. B. ohne weiteres vorstellbar, dass auch in den von den Autoren genannten und relativ zu den übrigen EU-Ländern wirtschaftlich ,zurückgebliebenen' baltischen Staaten, in denen sich gegenwärtig - nachdem der sowjetische Sozialismus egalitaristische und Solidaritätswerte bis zum Überdruss strapaziert hat - die Stimmung gänzlich auf Wachstumsziele konzentriert, die früher dominanten Wertperspektiven, sobald die ersten Etappen auf dem angezielten Wachstumspfad bewältigt sind, erneut in den Vordergrund treten.

In der Gerechtigkeitstheorie sind mehrere Möglichkeiten erkundet worden, wie man die Vielfalt der Gerechtigkeitsbegriffe und Gerechtigkeitskriterien respektieren und zugleich eindeutige Entscheidungsgrundlagen für soziale Verteilungen entwickeln kann. Eine ist die Zuordnung verschiedener Gerechtigkeitsbegriffe zu gesellschaftlichen Funktionsbereichen, wie sie Michael Walzer in seinem Buch Spheres of Justice (Walzer 1983) und Rainer Forst in Kontexte der Gerechtigkeit (Forst 1994) versucht haben. Eine solche Kontextualisierung der Gerechtigkeit kann sich darauf stützen, dass wir in unterschiedlichen Kontexten unterschiedliche Kriterien als plausibel empfinden. Die Bevorzugung von Familienangehörigen ist bei Entscheidungen darüber, wem man sein Vermögen vermacht, mehr oder weniger selbstverständlich, keineswegs aber bei der Vergabe von Ämtern oder Preisen. Leistungskriterien gelten bei der Rente als ,natürlich und angemessen, nicht aber bei der Sozialhilfe. Zahlungsfähigkeit ist beim Luxuskonsum, etwa beim Erwerb kostspieliger Autos, als Verteilungskriterium akzeptiert, nicht aber bei Gesundheitsdienstleistungen, zumindest nicht solchen, die über die Lebenserwartung entscheiden. Allerdings lassen sich die zwischen den einzelnen Gerechtigkeitskriterien aufbrechenden Konflikte durch eine Zuordnung zu Kontexten nur unvollständig aufösen. Nicht nur ist die Zuordnung von Kri- 
terien zu Bereichen keineswegs unumstritten. Ob etwa die Gesundheitsfürsorge stärker nach Prinzipien der Bedürfnisgerechtigkeit oder nach Marktprinzipien (oder auch nach Verdienstprinzipien) organisiert werden soll, ist gerade in der Gesellschaft der USA, auf die Walzers Konzeption zielt, hochgradig kontrovers. Auch ist für ein und denselben Bereich in der Regel nicht nur ein, sondern wiederum eine Vielfalt von untereinander unvereinbaren Kriterien relevant. Auch für eng definierte Kontexte ergeben sich ähnliche Konflikte und ähnliche Notwendigkeiten eines ,Mix‘ aus mehreren Kriterien wie auf der Ebene der Gesellschaft.

Ein einschlägiges Beispiel ist die seit Jahrzehnten anhaltende Kontroverse um die Kriterien der Verteilung knapper Organtransplantate. Auch hier bieten die Gerechtigkeitskriterien das an Hartmanns Wertrealismus erinnernde Bild einer gleichbleibenden Konstellation, auf die lediglich wechselnde Perspektiven projiziert werden. Was als ,gerecht' gilt, resultiert jeweils aus einer komplexen Mischung verschiedener untereinander unvereinbarer Gerechtigkeitskriterien. Betrachtet man jeweils ein Gerechtigkeitskriterium für sich, erscheint es wohlbegründet und plausibel. Versucht man jedoch, mehrere dieser Kriterien zu kombinieren und die jeweiligen Kombinationen in Prioritätslisten und Bewertungsalgorithmen umzusetzen, wird deutlich, wie eng die Spielräume für eine solche ,Mischung' - die ihrerseits wiederum plausibel sein soll - beschaffen sind.

Die besondere Schärfe des Konflikts zwischen den beteiligten Verteilungskriterien resultiert daraus, dass sich für jedes einzelne gewichtige und in unserem moralischen Denken gut verankerte moralische Begründungsprinzipien angeben lassen. So kann das Kriterium der Chancengleichheit, nach dem jeder Patient grundsätzlich das gleiche Recht hat, ein knappes Transplantat zu erhalten, auf die egalitaristische Idee der Menschenwürde zurückgeführt werden, die jedem Menschen in genau demselben Maße und ungeachtet seiner wie immer gearteten Verdienste und Aussichten zukommt. Das damit konkurrierende Kriterium der besonderen Berücksichtigung der Länge der Wartezeit entspricht dem traditionellen Prinzip der ausgleichenden Gerechtigkeit, nach dem diejenigen ein größeres Anrecht haben, die sich bestimmte Verdienste erworben, mehr Opfer gebracht oder mehr als andere gelitten haben. Geradezu konträr dazu steht das Kriterium der Verteilungseffizienz, nach dem diejenigen mit der größeren Erfolgsaussicht bevorzugt werden und das utilitaristischen Denkmustern entspricht. Beide Prinzipien sind im Gegensatz zum egalitaristischen Prinzip der Chancengleichheit differenzierende Prinzipien. Aber ansonsten stehen sich beide wie die zwei Seiten eines Januskopfes gegenüber: Das Prinzip der ausgleichenden Gerechtigkeit berücksichtigt ausschließlich die Vergangenheit, das Prinzip der Verteilungseffizienz ausschließlich die Zukunft. Und schließlich konkurrieren alle diese Kriterien mit einem vierten, das man vielleicht Mitleids-Kriterium nennen kann, nach dem der relativ Bedürftigste bevorzugt werden sollte, und zwar ungeachtet dessen, wie weit er selbst zu seiner Bedürftigkeit beigetragen hat und welche Aussichten er hat, von dieser Bevorzugung zu profitieren. Im Gegensatz zum Ausgleichsund Erfolgskriterium ist dieses Kriterium ausschließlich gegenwartsorientiert und wird von vielen mit dem spezifisch christlichen Motiv der Barmherzigkeit in Verbindung gebracht. Rawls' ,Differenzprinzip‘, das Gaertner und Schwettmann in den Mittelpunkt stellen, lässt sich als Variante dieses Mitleids-Kriteriums deu- 
ten: Die Gerechtigkeit eines sozialen Arrangements hängt danach primär oder ausschließlich davon ab, wie die jeweils Schlechtestgestellten bzw. die jeweils schlechtestgestellte Gruppe durch dieses Arrangement gestellt wird.

Für den Ethiker kommt das Hauptergebnis von Gaertners und Schwettmanns Untersuchung, die Abhängigkeit der Priorisierung des Differenzprinzips von wechselnden kulturellen, ökonomischen und politischen Faktoren, nicht überraschend. Das Rawlssche Differenzprinzip ist unter allen Gerechtigkeitskriterien möglicherweise dasjenige, dessen Plausibilität am weitgehendsten von den kontingenten Rahmenbedingungen seiner Anwendung abhängt und am empfindlichsten auf Wandlungen in diesen Rahmenbedingungen reagiert. Abzuhängen scheint die Plausibilität des Differenzprinzips von mindestens drei Faktoren: 1. dem absoluten Wohlstandsniveau der jeweils schlechtestgestellten Gruppe; 2. dem Ausmaß, in dem für Umverteilungen zugunsten der jeweils schlechtestgestellten Gruppe Wohlstandseinbußen der in der Regel zahlenmäßig größeren Gruppe der relativ Bessergestellten in Kauf genommen werden müssen; und 3. dem Ausmaß, in dem die Gesamtproduktivität der Gesellschaft durch eine Umverteilung im Sinne des Differenzprinzips beeinträchtigt wird. Je höher das absolute Wohlstandsniveau der relativ Schlechtgestellten über dem sozialen Existenzminimum liegt, desto weniger scheint für die Regel zu sprechen, die Gruppe der relativ Schlechtestgestellten zuungunsten aller anderen Gruppen einschließlich der Gruppe der unter den übrigen Gruppen Schlechtestgestellten - zu priorisieren. Dasselbe scheint zu gelten, je einschneidender die Einbußen sind, die der zahlenmäßig größeren Gruppe der relativ Bessergestellten zugunsten der schlechtestgestellten auferlegt werden müssen. Und schließlich scheinen auch Überlegungen der Gesamtproduktivität für die intuitive Plausibilität von Gerechtigkeitskriterien eine Rolle zu spielen: Je stärker in Bezug auf bestimmte Güter (potenziell) produktive Gruppen durch eine Anwendung des Kriteriums gegenüber einem Anfangszustand schlechtergestellt werden, desto weniger akzeptabel erscheint das Kriterium, insbesondere bei längerfristiger Betrachtung.

Man wird sofort bemerken, dass sich in diesen Intuitionen Gerechtigkeitsprinzipien geltend machen, die durch das Differenzprinzip nicht oder nicht ausreichend erfasst werden: einerseits utilitaristische Gesichtspunkte der zukunftsbezogenen Maximierung, andererseits Gesichtspunkte der ausgleichenden Gerechtigkeit, zumindest insoweit, als die Bessergestellten ihre Besserstellung im Sinne eines option luck primär oder ausschließlich eigenen Anstrengungen und nicht dem brute luck der natürlichen und sozialen Lotterie verdanken. Was die Untersuchung nahelegt, ist, dass das Ausmaß, in dem diese abweichenden bzw. zusätzlichen Kriterien eine Rolle für die Auffassungen von Gerechtigkeit spielen, von den Zeitumständen beeinflusst sind. In einer Zeit, in der nach einer längeren Latenzphase erneut die Themen Wirtschaftswachstum und ,Wachstumsschwäche die Schlagzeilen und die politische Agenda dominieren, ist auch von den Gerechtigkeitsauffassungen ein bias in diese Richtung zu erwarten.

Empirische Ergebnisse dieser Art sollten nicht nur in der Politik, sondern auch in der Ethik zur Kenntnis genommen werden. Immerhin verfolgen ethische Arbeiten zur Gerechtigkeit in der Regel nicht nur theoretische, sondern auch im weitesten Sinne praktische Ziele. Sie wollen die faktische Urteils- und Ent- 
scheidungspraxis nicht nur analysieren oder begründen, sondern zumeist auch im Sinne bestimmter Prinzipien beeinflussen. Jeder Versuch einer Beeinflussung dieser Praxis wird jedoch solange scheitern, wie sie nicht die empirisch aufweisbaren Strukturen dieser Praxis und die innerhalb der Sozialwissenschaften vorgeschlagenen Hypothesen und Theorien zu deren wahrscheinlichen Verursachungsfaktoren berücksichtigt. Soweit die Ethik nicht nur Theorie bleiben will, sondern in die von ihr in abstracto diskutierten Denkweisen auch praktisch eingreifen will, ist sie notwendig auf empirische Daten und deren fachkundige Interpretation angewiesen (vgl. Birnbacher 1999). Wenn sie von der bloßen Theorie zu dem übergehen will, was Kant zu Anfang seiner Abhandlung Über den Gemeinspruch: Das mag in der Theorie richtig sein, taugt aber nicht für die Praxis ,wahre Theorie' genannt hat: eine nicht nur in ihren ethischen Prinzipien stimmige, sondern auch hinsichtlich ihres Erfahrungsgehalts angemessene und erst dadurch für die Praxis taugliche Theorie, bedarf sie notwendig der Sozialwissenschaften. Kant jedenfalls zögerte nicht, dem abstrakten und bloß in Prinzipien handelnden Theoretiker den Vorwurf zu machen: „Da lag es dann nicht an der Theorie, wenn sie zur Praxis noch wenig taugte, sondern daran, daß nicht genug Theorie da war, welche der Mann von der Erfahrung hätte lernen sollen." (Kant, $1793 / 1923,275)$

\section{Bibliographie}

Birnbacher, D. (1999), Ethics and Social Science: Which Kind of Co-operation? in: Ethical Theory and Moral Practice 2, 319-336

Forst, R. (1994), Kontexte der Gerechtigkeit, Frankfurt

Kant, I. (1923) „Über den Gemeinspruch: Das mag in der Theorie richtig sein, taugt aber nicht für die Praxis", in Kants Werke, Akademie-Ausgabe Bd. 8, Berlin, 273314

Walzer, M. (1983), Spheres of Justice. A Defense of Pluralism and Equality, New York 